\title{
Collective Oscillations of Majorana Neutrinos in Strong Magnetic Fields and Self-induced Flavor Equilibrium
}

\author{
Sajad Abbar ${ }^{1,2}$ \\ ${ }^{1}$ Max-Planck-Institut für Physik (Werner-Heisenberg-Institut), \\ Föhringer Ring 6, 80805 München, Germany \\ ${ }^{2}$ Astro-Particule et Cosmologie (APC), CNRS UMR 7164, \\ Université Denis Diderot, 75205 Paris Cedex 13, France
}

\begin{abstract}
We study collective oscillations of Majorana neutrinos in some of the most extreme astrophysical sites such as neutron star merger remnants and magneto-rotational core-collapse supernovae which include dense neutrino media in the presence of strong magnetic fields. We show that neutrinos can reach flavor equilibrium if neutrino transition magnetic moment $\mu_{\nu}$ is strong enough, namely when $\mu_{\nu} / \mu_{\mathrm{B}} \gtrsim 10^{-14}-10^{-15}$ with $\mu_{\mathrm{B}}$ being the Bohr magneton. This sort of flavor equilibrium, which is not necessarily flavor equipartition, can occur on (short) scales determined by the strength of the magnetic term in the Hamiltonian. Our findings can have interesting implications for the physics of such violent astrophysical environments.
\end{abstract}

\section{INTRODUCTION}

Neutrinos can play a vital role in the physics of the most violent astrophysical phenomena such as neutron star mergers (NSM) and core-collapse supernovae (CC$\mathrm{SNe}$ [1-4]. Due to their weak interactions, they can act as the major channel of energy transport. Moreover, they can be crucial to heavy elements nucleosynthesis since they can modify the neutron to proton ratio through the weak reactions $\bar{\nu}_{e}+p \rightleftharpoons n+e^{+}$and $\nu_{e}+n \rightleftharpoons p+e^{-}[5]$.

Neutrinos can experience flavor conversions which can change their energy spectra. This, in principle, can change their interaction rates and consequetly influence their effects on the dynamics and nucleosynthesis in the extreme astrophysical environments. In addition, on the observational side, any flavor conversions can modify the neutrino signal which may be observed from these events on the earth.

Neutrinos can experience collective flavor oscillations in NSM remnants and CCSNe due to their coherent forward scatterings by the high density background neutrino gas. The presence of neutrino-neutrino interaction makes the problem of neutrino evolution in a dense neutrino medium very demanding and remarkably different from the one in vacuum and matter. It, indeed, makes this problem a nonlinear one with strong coupling among different neutrino momenta [6-10].

The first studies on this problem where carried out in maximally symmetric models. For example, to study collective neutrino oscillations in the supernova context, a stationary spherically symmetric SN model, i.e. the socalled neutrino bulb model was used [7]. The most important feature of the results obtained in the bulb model is the presence of the spectral swapping phenomenon in which $\nu_{e}\left(\bar{\nu}_{e}\right)$ exchanges its spectra with $\nu_{x}\left(\bar{\nu}_{x}\right)$ for a certain range of neutrino energies $[8,9,11-15]$. This phenomenon is a direct consequence of collective neutrino oscillations.

However, regarding the evolution of neutrinos in NSM remnant accretion disks, the geometry is much more com- plicated and a self-consistent one-dimensional model is unavailable. The first studies were done in the so-called single-angle approximation scenario [16] in which it is assumed that all neutrinos emitted from the neutrino emitting accretion disk experience similar flavor evolution. The salient characteristic of the results obtained in these calculations is the occurrence of matter-neutrino resonance (MNR) [17-26]. This phenomenon results from the cancellation between the neutrino-neutrino interaction and matter potentials which can happen since in the NSM environment, $\bar{\nu}_{e}$ can be more abundant than $\nu_{e}$, i.e. $n_{\bar{\nu}_{e}} / n_{\nu_{e}}>1$. The MNR phenomenon is currently thought to be absent in the supernova environment where normally $n_{\bar{\nu}_{e}} / n_{\nu_{e}}<1$ and as a result, the neutrino and matter potentials have similar signs ${ }^{1}$.

Nevertheless, it was then realised that such oversimplified maximally symmetric models are not appropriate to study neutrino flavor evolution in dense neutrino media. On the one hand, the spatial and time symmetries in the neutrino gas can be broken spontaneously in the presence of collective neutrino oscillations [10,30-39]. This can allow for neutrino flavor conversions at very large matter/neutrino densities. On the other hand, it has been shown that neutrinos can experience the so-called fast flavor conversion modes in dense neutrino media probably provided that $\nu_{e}$ and $\bar{\nu}_{e}$ angular distributions cross each other [38, 40-56]. Such fast conversion modes can occur on scales $\sim G_{\mathrm{F}}^{-1} n_{\nu}^{-1}$ which can be as short as a few $\mathrm{cm}$ 's in the aforementioned extreme astrophysical environments. This must be compared with slow modes expected to occur on scales $\sim \mathcal{O}(1) \mathrm{km}$ (for a $10 \mathrm{MeV}$ neutrino) determined by the neutrino vacuum frequency $\omega=\Delta m_{\mathrm{atm}}^{2} / 2 E$.

\footnotetext{
1 Note that there can exist SN zones inside the proto-neutron star for which $n_{\bar{\nu}_{e}} / n_{\nu_{e}}>1$ [27-29]. Despite this, the matter density is much larger than the neutrino number densities at these zones and the cancelation between the neutrino-neutrino interaction and matter potentials seems to be inacssisible.
} 
In addition, neutrinos are expected to have tiny but nonzero magnetic moments (see, e.g., [57-59] for a review) which can influence their flavor evolution in the presence of magnetic fields. In particular, the presence of ultra-strong magnetic fields $\left(B \gtrsim 10^{15}\right.$ Gauss [60]) in NSM and magneto-rotational CCSNe (in which rapid rotation and large magnetic fields are thought to play an important role) makes them ideal settings for studying the impact of the coupling between neutrinos and magnetic field (photon) on collective neutrino oscillations. While such a coupling leads to active-sterile neutrino oscillations in the case of Dirac neutrinos, it results in neutrino-antineutrino oscillations for Majorana neutrinos.

In the minimally-extended Standard Model (MESM), the diagonal magnetic moment of Dirac neutrinos can be written as [61]

$$
\mu_{\nu}^{i i, D}=\frac{3 e G_{\mathrm{F}} m_{\nu}}{8 \sqrt{2} \pi^{2}} \simeq 3.2 \times 10^{-19}\left(\frac{m_{\nu}}{1 \mathrm{eV}}\right) \mu_{\mathrm{B}},
$$

where $m_{\nu}$ is the neutrino mass and $\mu_{\mathrm{B}}=5.788 \times 10^{-9}$ $\mathrm{eV}$ Gauss $^{-1}$ is the Bohr magneton. The transition magnetic moment is smaller than the diagonal one by approximately four orders of magnitude. As for Majorana neutrinos, while the diagonal magnetic moment is dictated to be zero, the transition magnetic moment is similar to the transition magnetic moment of Dirac neutrinos.

Although MESM predicts $\mu_{\nu} \lesssim 10^{-19} \mu_{\mathrm{B}}$, some of the theories beyond SM predict (or at least can explain) much larger values for $\mu_{\nu}{ }^{2}$. In fact, the current experiments can only provide an upper bound on $\mu_{\nu}$ (see, e.g., Refs. $[65,66])$,

$$
\mu_{\nu} \lesssim 3 \times 10^{-11} \mu_{\mathrm{B}}
$$

which is many orders of magnitude larger than the value suggested by MESM. This constraint is valid for both Dirac and Majorana neutrinos and also diagonal and transition magnetic moments.

The coupling between neutrinos and magnetic field can provide new channels for changing neutrino lepton number and can possibly lead to new physics, if it is strong enough. A number of papers have studied this phenomenon in astrophysical environment [67-82]. In particular, in Refs. [78, 79] the authors reported that

\footnotetext{
2 There can indeed exist some difficulties here [62-64]. In particular, since the neutrino magnetic moment can depend linearly on the neutrino mass, any attempt to increase the neutrino magnetic moment leads to an increase in the neutrino mass as well. Thus, to be consistent with current constraints on the neutrino mass and at the same time having large values for $\mu_{\nu}$, one may need a sort of fine-tuning. Though for Dirac neutrinos this necessary fine-tuning leads to theoretical difficulties to produce $\mu_{\nu} \gtrsim 10^{-15} \mu_{\mathrm{B}}$, it is almost harmless to the case of Majorana neutrinos since it only becomes problematic when $\mu_{\nu} \gtrsim 10^{-9} \mu_{\mathrm{B}}$ which is already excluded by experiments.
}

collective oscillations of Majorana neutrinos can be nontrivially affected by the magnetic term for level-of-SM or even smaller $\mu_{\nu}$ 's.

In this paper, we study collective oscillations of Majorana neutrinos in the presence of strong magnetic fields with $B \gtrsim 10^{15}$ Gauss, thought to be present in NSM remnants and magneto-rotational CCSNe. To achieve this goal, we use a schematic multi-angle one-dimensional model for the neutrino gas in the two-flavor (Sec. II A) and three-flavor (Sec. IIB) scenarios. We show that if the neutrino magnetic moment is large enough, the neutrino gas can reach a sort of flavor equilibrium (which is not necessarily equipartition) on scales determined by the magnetic term.

\section{COLLECTIVE OSCILLATIONS OF MAJORANA NEUTRINOS IN THE PRESENCE OF MAGNETIC FIELDS}

To study the evolution of Majorana neutrinos in the presence of strong magnetic fields, we consider a singleenergy, multi-angle neutrino gas in both two and threeflavor scenarios in which neutrinos are emitted with emission angles in the range $\left[-\vartheta_{\max }, \vartheta_{\max }\right]$. This model is similar to the one used in Ref. [49].

At each space-time point $(t, \mathbf{r})$, the flavor state of a neutrino traveling in direction $\vartheta$ can be specified by its density matrix $\rho_{\vartheta}(t, \mathbf{r})$. The evolution of $\rho_{\vartheta}(t, \mathbf{r})$ in the absence of collisions is governed by the Liouville-von Neumann equation of motion [83-87]

$$
i \mathrm{D}_{\mathrm{t}} \rho_{\vartheta}=\left[\mathrm{H}_{\vartheta}, \rho_{\vartheta}\right],
$$

where $\mathrm{D}_{\mathrm{t}}=\partial_{\mathrm{t}}+\mathbf{v} \cdot \nabla$ and $\mathrm{H}_{\vartheta}=\mathrm{H}_{\mathrm{vac}}+\mathrm{H}_{\text {mat }}+\mathrm{H}_{\nu \nu, \vartheta}$ is the total Hamiltonian, with $\mathrm{H}_{\mathrm{vac}}, \mathrm{H}_{\text {mat }}$ and $\mathrm{H}_{\nu \nu, \vartheta}$ being the contributions from vacuum, matter and neutrinoneutrino interaction potentials, respectively. Here, the contribution from the coupling between neutrinos and magnetic field is included in the vacuum term.

In our study, the evolution of neutrinos is considered in two models, namely a stationary one-dimensional model and a time-dependent homogenous neutrino gas. In the one-dimensional model $\mathrm{D}_{\mathrm{t}}=\cos \vartheta \mathrm{d}_{\mathrm{r}}$ while one has $D_{t}=d_{t}$ in the time-dependent homogenous gas. As will be seen in what follows, the occurrence and nature of the equilibrium does not depend on the employed model since the outcome is purely determined by the presence of the strong magnetic coupling term. Nevertheless, the amplitude of the oscillations around the equilibrium can be smaller in the stationary one-dimensional model. We also assume that the physical quantities such as the matter/neutrino densities and magnetic field are constant during the propagation of neutrinos. This is justified by noting that the scales associated with neutrino oscillations in this problem (induced by strong magnetic coupling) are much shorter than the relevant scales of the astrophysical problems of interest. 


\section{A. Two-flavor scenario}

To demonstrate the idea and to show how the presence of strong coupling between neutrinos and magnetic field can influence their oscillations in a dense neutrino medium, we first start with the case of two-flavor scenario. We follow the formalism developed in Ref. [78, 79] and we take $\rho$ to be a $4 \times 4$ matrix which includes the flavor content of neutrinos and antineutrinos

$$
\rho=\left[\begin{array}{llll}
\rho_{\nu_{e} \nu_{e}} & \rho_{\nu_{e} \nu_{x}} & \rho_{\nu_{e} \bar{\nu}_{e}} & \rho_{\nu_{e} \bar{\nu}_{x}} \\
\rho_{\nu_{x} \nu_{e}} & \rho_{\nu_{x} \nu_{x}} & \rho_{\nu_{x}} \bar{\nu}_{e} & \rho_{\nu_{x} \bar{\nu}_{x}} \\
\rho_{\bar{\nu}_{e} \nu_{e}} & \rho_{\bar{\nu}_{e} \nu_{x}} & \rho_{\bar{\nu}_{e} \bar{\nu}_{e}} & \rho_{\bar{\nu}_{e} \bar{\nu}_{x}} \\
\rho_{\bar{\nu}_{x} \nu_{e}} & \rho_{\bar{\nu}_{x} \nu_{x}} & \rho_{\bar{\nu}_{x}} \bar{\nu}_{e} & \rho_{\bar{\nu}_{x} \bar{\nu}_{x}}
\end{array}\right]
$$

where the diagonal terms are basically the occupation numbers of the corresponding neutrino flavors and offdiagonal terms carry information on neutrino flavor mixing. This matrix has clearly the form

$$
\rho=\left[\begin{array}{cc}
\rho_{\nu} & X \\
X^{\dagger} & \rho_{\bar{\nu}}
\end{array}\right]
$$

with

$$
X=\left[\begin{array}{ll}
\rho_{\nu_{e} \bar{\nu}_{e}} & \rho_{\nu_{e} \bar{\nu}_{x}} \\
\rho_{\nu_{x} \bar{\nu}_{e}} & \rho_{\nu_{x} \bar{\nu}_{x}}
\end{array}\right],
$$

and $\rho_{\nu}$ and $\rho_{\bar{\nu}}$ being the usual $2 \times 2$ flavor matrices having information on the flavor content of neutrinos and antineutrinos, respectively. It is very convenient to follow this formalism here since for nonzero Majorana neutrino magnetic moment, neutrinos and antineutrinos are coupled in the presence of magnetic field and there is a nonzero $\nu-\bar{\nu}$ transition amplitide.

Within this formalism, the vacuum and matter potentials can be written as

$\mathrm{H}_{\mathrm{vac}}=\left[\begin{array}{cccc}-\omega \cos 2 \theta_{\mathrm{v}} & \omega \sin 2 \theta_{\mathrm{v}} & 0 & \Omega \\ \omega \sin 2 \theta_{\mathrm{v}} & \omega \cos 2 \theta_{\mathrm{v}} & -\Omega & 0 \\ 0 & -\Omega & -\omega \cos 2 \theta_{\mathrm{v}} & \omega \sin 2 \theta_{\mathrm{v}} \\ \Omega & 0 & \omega \sin 2 \theta_{\mathrm{v}} & \omega \cos 2 \theta_{\mathrm{v}}\end{array}\right]$,

$\mathrm{H}_{\text {mat }}=\left[\begin{array}{cccc}\left(\lambda_{e}-\lambda_{n} / 2\right) & 0 & 0 & 0 \\ 0 & -\lambda_{n} / 2 & 0 & 0 \\ 0 & 0 & -\left(\lambda_{e}-\lambda_{n} / 2\right) & 0 \\ 0 & 0 & 0 & \lambda_{n} / 2\end{array}\right]$,

where $\lambda_{\mathrm{e}(\mathrm{n})}=\sqrt{2} G_{\mathrm{F}} n_{e(n)}$, with $n_{e}\left(n_{n}\right)$ being the electron (neutron) number density and $\theta_{\mathrm{v}}$ and $\omega=$ $\Delta m_{\mathrm{atm}}^{2} / 2 E$ are the neutrino vacuum mixing angle and the vacuum frequency $\left(\Delta m_{\mathrm{atm}}^{2}>0(<0)\right.$ for the normal (inverted) mass hierarchy) for a neutrino with energy $E$. In our calculations, we set $\theta_{\mathrm{v}}=0.1$ and $\omega=1$ though the results do not qualitatively depend on the choice of these parameters. Note that the vacuum term has the new contribution $\Omega=\mu_{\nu} B_{\mathrm{T}}$ from the coupling of Majorana neutrino with the component of magnetic field transverse to the neutrino momentum, $B_{\mathrm{T}}$. Furthermore, unlike the case of collective neutrino oscillations in the absence of magnetic field, the neutral current contribution from neutrons to the matter potential can not be ignored since it has different signs for neutrinos and antineutrinos and can not be removed as a common phase when these two are coupled.

In addition, the neutrino-neutrino interaction potential, $\mathrm{H}_{\nu \nu, \vartheta}$, is ${ }^{3}$

$$
\begin{aligned}
\mathrm{H}_{\nu \nu, \vartheta} & =\sqrt{2} G_{\mathrm{F}} \int_{-\vartheta_{\max }}^{\vartheta_{\max }} \mathrm{d} \vartheta^{\prime}\left(1-\cos \left(\vartheta-\vartheta^{\prime}\right)\right) \\
& \times\left[G^{\dagger}\left(\rho_{\vartheta^{\prime}}-\rho_{\vartheta^{\prime}}^{c *}\right) G+\frac{1}{2} G^{\dagger} \operatorname{tr}\left(\left(\rho_{\vartheta^{\prime}}-\rho_{\vartheta^{\prime}}^{\mathrm{c*}}\right) \mathrm{G}\right)\right]
\end{aligned}
$$

where

$$
G=\left[\begin{array}{cccc}
+1 & 0 & 0 & 0 \\
0 & +1 & 0 & 0 \\
0 & 0 & -1 & 0 \\
0 & 0 & 0 & -1
\end{array}\right]
$$

and $\rho^{c}$ is defined as

$$
\rho^{c}=\left[\begin{array}{cc}
\rho_{\bar{\nu}} & X^{*} \\
X^{T} & \rho_{\nu}
\end{array}\right] .
$$

Note that the definition of $\rho^{c}$ is somewhat different from the one in Refs. [78, 79] so that there is no contribution to $\nu-\bar{\nu}$ transition from the neutrino-neutrino interaction term $[80,88]$ ( see also $[87,89,90])$. The last term in Eq. (9) refers to a phase factor which has different signs for neutrinos and antineutrinos and therefore, can not be removed here. One can then recover the usual equations of motion of traditional collective oscillations if $B=0$.

\section{Results}

In our simulations, we took $\vartheta_{\max }=\pi / 3$ and a fixed magnetic field with $B_{\mathrm{T}}=5 \times 10^{15}$ Guass. Such strong magnetic fields may not exist on very large scales in the astrophysical problems of interest. However, the scales associated with neutrino oscillations for strong $\Omega$ 's are much shorter than other relevant scales in the problem and therefore, we here intend to consider the local effects of large $\Omega$ 's rather than the global ones. Thus, the physical quantities are assumed to be constant. Note also that since Hamiltonian is only sensitive to $B$ via $\mu_{\nu} B_{\mathrm{T}}$, for smaller/larger magnetic fields one can just rescale $\mu_{\nu}$.We also set $n_{\nu_{\mu}}=n_{\bar{\nu}_{\mu}}=n_{\nu_{\tau}}=n_{\bar{\nu}_{\tau}}=0.4 n_{\nu_{e}}$ in our calculations.

The angle-averaged neutrino survival probabilities of neutrinos and antineutrinos are shown in Figs. 1 and 2.

\footnotetext{
3 Here and in Eq. (8), we assume having a large $\mu_{\nu}$ does not modify neutrino weak interactions (similar to, e.g., Refs. [71, 72]).
} 

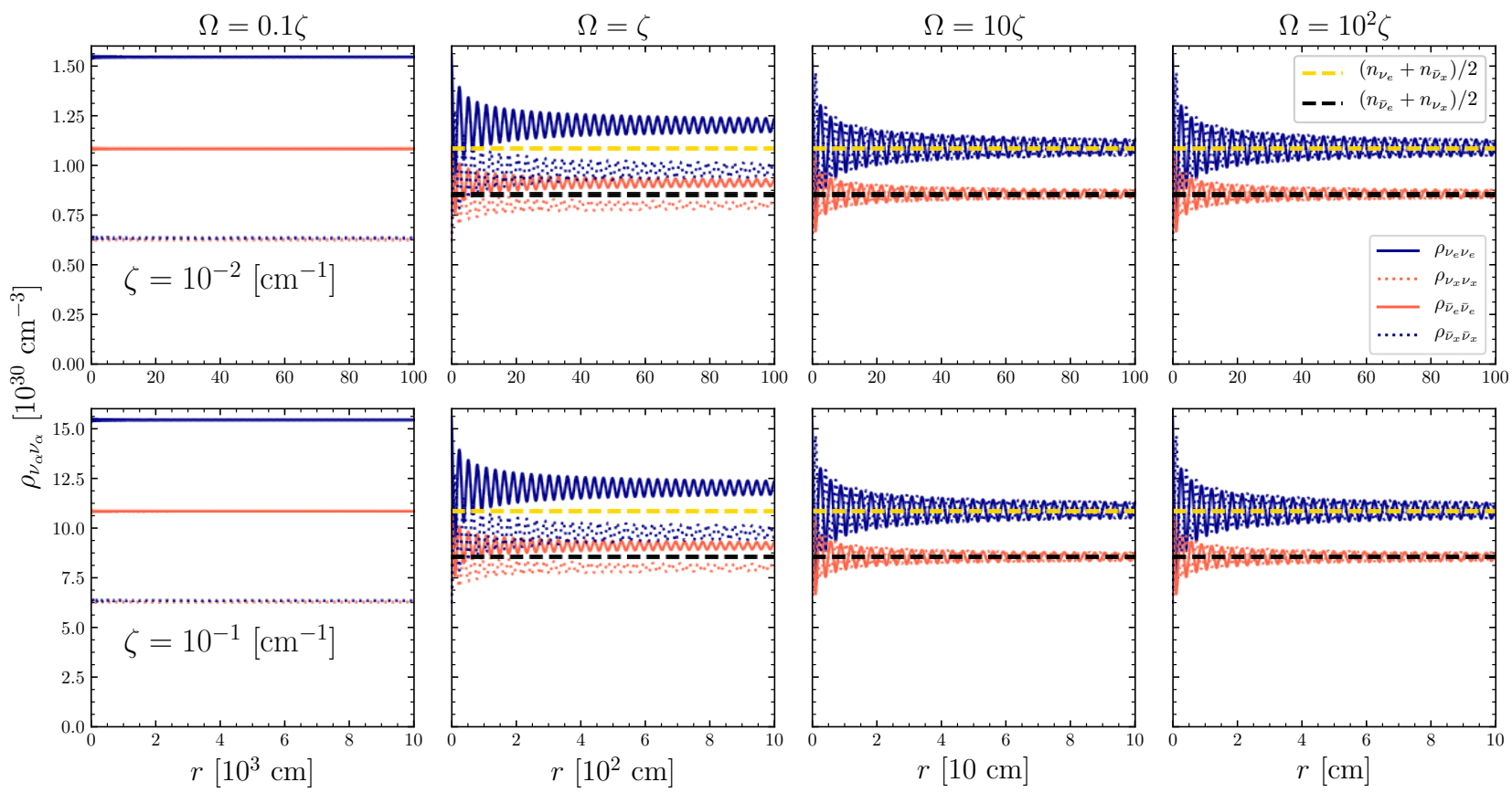

FIG. 1. Angle-averaged survival probabilities of neutrinos and antineutrinos for $n_{\bar{\nu}_{e}} / n_{\nu_{e}}=0.7$. The evolution of neutrinos is studied in the homogenous time-dependent model and as mentioned in the text, $\mu_{\nu}$ can be found from $\mu_{\nu}=6.8 \times 10^{-15} \mu_{\mathrm{B}}(\Omega / \zeta)\left(\zeta / 10^{-2} \mathrm{~cm}^{-1}\right)$ in each panel. Note that the neutrino oscillations scale is $\sim 1 / \Omega$ for strong $\Omega$ 's.
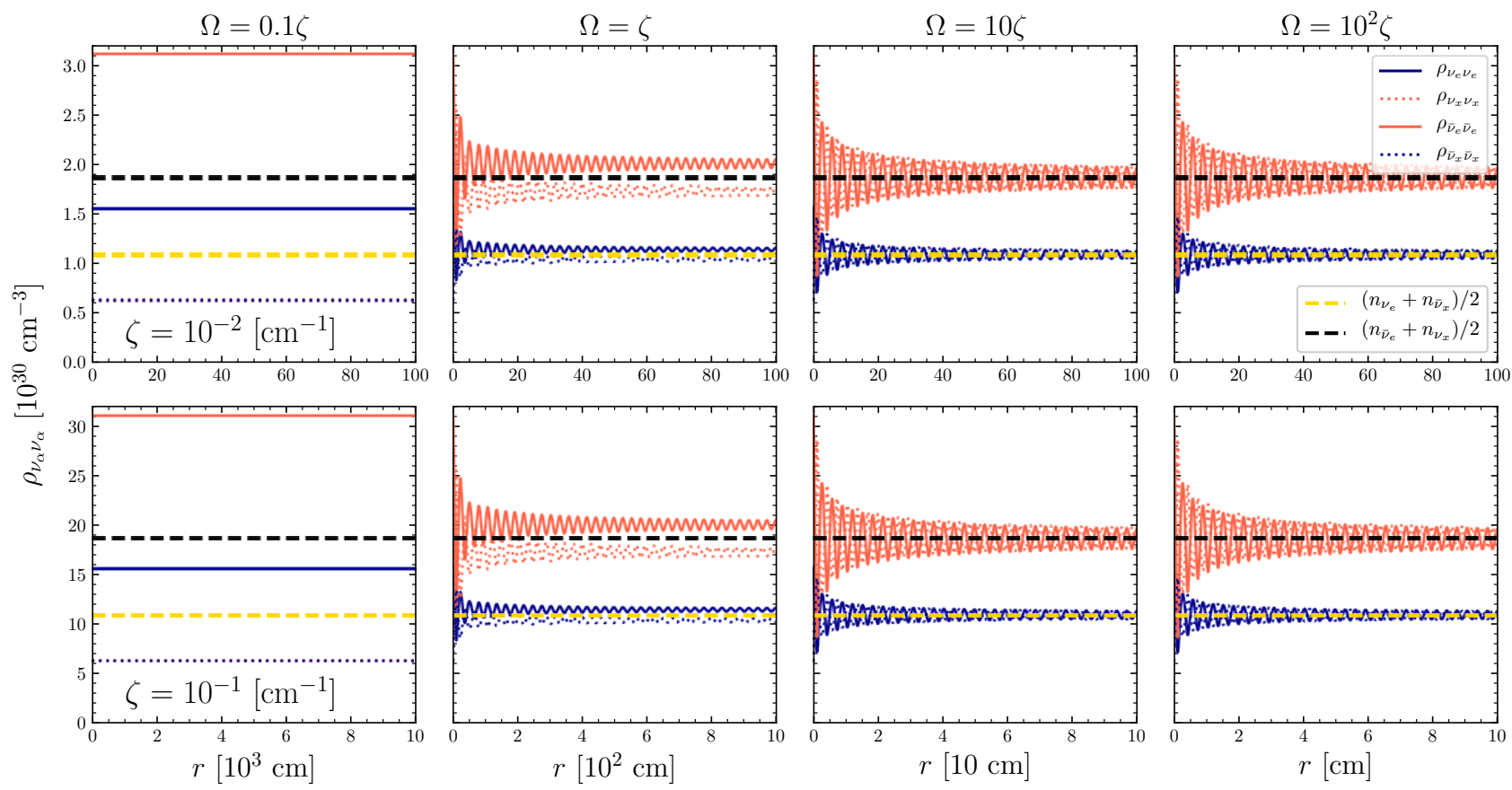

FIG. 2. The same information as in Fig. 1 for $n_{\bar{\nu}_{e}} / n_{\nu_{e}}=2$.

We considered two cases with $n_{\bar{\nu}_{e}} / n_{\nu_{e}}=0.7$ and 2 , for a number of $\Omega$ 's and two neutrino number densities speci- fied by

$$
\zeta=\sqrt{2} G_{\mathrm{F}} n_{\nu_{e}}
$$


For each panel, the corresponding neutrino magnetic moment is

$$
\mu_{\nu}=6.8 \times 10^{-15} \mu_{\mathrm{B}}\left(\frac{\Omega}{\zeta}\right)\left(\frac{\zeta}{10^{-2} \mathrm{~cm}^{-1}}\right) .
$$

We have confirmed that our results do not qualitatively depend on the choice of $n_{\bar{\nu}_{e}} / n_{\nu_{e}}$ and $n_{\nu_{x}} / n_{\nu_{e}}$, as well as electron and neutron densities (as long as $\Omega$ is the dominant term) and the mass term in the Hamiltonian.

As can be clearly seen in Figs. 1 and 2, the magnetic term does not noticeably modify neutrino flavor evolution for small $\Omega$ 's $(\Omega \lesssim 0.5 \zeta)$. But if $\Omega$ term is comparable to the rest of the Hamiltonian (here dominated by $\mathrm{H}_{\nu \nu}$ ), the neutrino gas experiences an interesting sort of flavor equilibrium in which $\nu_{e}\left(\bar{\nu}_{e}\right)$ reaches an approximate equalisation with $\bar{\nu}_{x}\left(\nu_{x}\right)$ so that

$$
\begin{aligned}
& \rho_{\nu_{e} \nu_{e}} \simeq \rho_{\bar{\nu}_{x} \bar{\nu}_{x}} \simeq \frac{n_{\nu_{e}}+n_{\bar{\nu}_{x}}}{2} \\
& \rho_{\bar{\nu}_{e} \bar{\nu}_{e}} \simeq \rho_{\nu_{x} \nu_{x}} \simeq \frac{n_{\bar{\nu}_{e}}+n_{\nu_{x}}}{2}
\end{aligned}
$$

with some small amplitude oscillations around the equilibrium value which can become smaller for $\Omega>$ $\sqrt{2} G_{\mathrm{F}} n_{\nu_{e}}$. The from of flavor equilibrium is indeed independent of the ratio $n_{\bar{\nu}_{e}} / n_{\nu_{e}}$.

The special form of the equilibrium arises from the specific structure of the vacuum Hamiltonian which couples $\nu_{e} \leftrightarrow \bar{\nu}_{x}$ and $\bar{\nu}_{e} \leftrightarrow \nu_{x}$. For strong $\Omega$ 's, the vacuum term dominates the evolution of neutrinos meaning that the neutrino oscillations is caused and triggered by the magnetic term which implies that the neutrino oscillation frequency is here determined by $\Omega$. This, combined with the decoherence induced by the neutrino-neutrino interaction term can then lead to the flavor equilibrium. Note that here the flavor conversion does not arise from cancellation between the diagonal terms in the Hamiltonian as in Refs. [69, 72], where resonant conversion is responsible for neutrino flavor oscillations.

\section{B. Three-flavor scenario}

Three-flavor oscillations of a dense neutrino gas in the presence of strong coupling between neutrinos and magnetic field can be studied as a straightforward generalisation of the two-flavor case.

The $6 \times 6$ neutrino density matrix

$$
\rho=\left[\begin{array}{cc}
\rho_{\nu} & X \\
X^{\dagger} & \rho_{\bar{\nu}}
\end{array}\right]
$$

includes the flavor contents of both neutrinos and antineutrinos of all three flavors with

$$
X=\left[\begin{array}{ccc}
\rho_{\nu_{e} \bar{\nu}_{e}} & \rho_{\nu_{e} \bar{\nu}_{\mu}} & \rho_{\nu_{e} \bar{\nu}_{\tau}} \\
\rho_{\nu_{\mu} \bar{\nu}_{e}} & \rho_{\nu_{\mu} \bar{\nu}_{\mu}} & \rho_{\nu_{\mu} \bar{\nu}_{\tau}} \\
\rho_{\nu_{\tau} \bar{\nu}_{e}} & \rho_{\nu_{\tau} \bar{\nu}_{\mu}} & \rho_{\nu_{\tau} \bar{\nu}_{\tau}}
\end{array}\right]
$$

and $\rho_{\nu}$ and $\rho_{\bar{\nu}}$ are the usual $3 \times 3$ flavor matrices of neutrinos and antineutrinos, respectively, defined as

$$
\rho=\left[\begin{array}{ccc}
\rho_{\nu_{e} \nu_{e}} & \rho_{\nu_{e} \nu_{\mu}} & \rho_{\nu_{e} \nu_{\tau}} \\
\rho_{\nu_{\mu} \nu_{e}} & \rho_{\nu_{\mu} \nu_{\mu}} & \rho_{\nu_{\mu} \nu_{\tau}} \\
\rho_{\nu_{\tau} \nu_{e}} & \rho_{\nu_{\tau} \nu_{\mu}} & \rho_{\nu_{\tau} \nu_{\tau}}
\end{array}\right]
$$

and similarly for antineutrinos.

In addition, the vacuum Hamiltonian can be written as

$$
\mathrm{H}_{\mathrm{vac}}=\left[\begin{array}{cc}
\tilde{H}_{\mathrm{vac}} & \tilde{H}_{\mathrm{B}} \\
-\tilde{H}_{\mathrm{B}} & \tilde{H}_{\mathrm{vac}}^{*}
\end{array}\right]
$$

where $\tilde{H}_{\text {vac }}$ is the usual $3 \times 3$ three-flavor vacuum Hamiltonian described by two mass-squared differences $\Delta m_{12}^{2}$ and $\Delta m_{13}^{2}$, three mixing angles $\theta_{12}, \theta_{13}$ and $\theta_{23}$, and one $C P$-violating phase $\delta^{4}$, for which the values were taken from Particle Data Group [91]. Also,

$$
\tilde{H}_{\mathrm{B}}=\left[\begin{array}{ccc}
0 & \Omega_{e \mu} & \Omega_{e \tau} \\
-\Omega_{e \mu} & 0 & \Omega_{\mu \tau} \\
-\Omega_{e \tau} & -\Omega_{\mu \tau} & 0
\end{array}\right]
$$

describes the contribution from the magnetic term where $\Omega_{\alpha \beta}=\mu_{\alpha \beta} B_{\mathrm{T}}$ are assumed to be real quantities. Moreover, in the neutrino-neutrino interaction term, Eq. (9), $G$ and $\rho^{c}$ are straightforward $6 \times 6$ generalisations of the corresponding $4 \times 4$ ones,

$$
G=\left[\begin{array}{cccccc}
+1 & 0 & 0 & 0 & 0 & 0 \\
0 & +1 & 0 & 0 & 0 & 0 \\
0 & 0 & +1 & 0 & 0 & 0 \\
0 & 0 & 0 & -1 & 0 & 0 \\
0 & 0 & 0 & 0 & -1 & 0 \\
0 & 0 & 0 & 0 & 0 & -1
\end{array}\right]
$$

and

$$
\rho^{c}=\left[\begin{array}{cc}
\rho_{\bar{\nu}} & X^{*} \\
X^{T} & \rho_{\nu}
\end{array}\right]
$$

\section{Results}

Collective neutrino oscillations in strong magnetic field in the three-flavor scenario is very similar to the one in the two-flavor scenario. In particular, the angle-averaged survival probabilities can reach some sort of flavor equilibrium, as indicated in Fig. 3. However, the magnetic term is more complicated in the three-flavor scenario. Thus, different flavors can, in general, reach different equilibrium values. For example, in our calculations with

\footnotetext{
4 Although we set $\delta=0$ in our calculations, we have confirmed that the results do not qualitatively depend on the choice of $\delta$.
} 

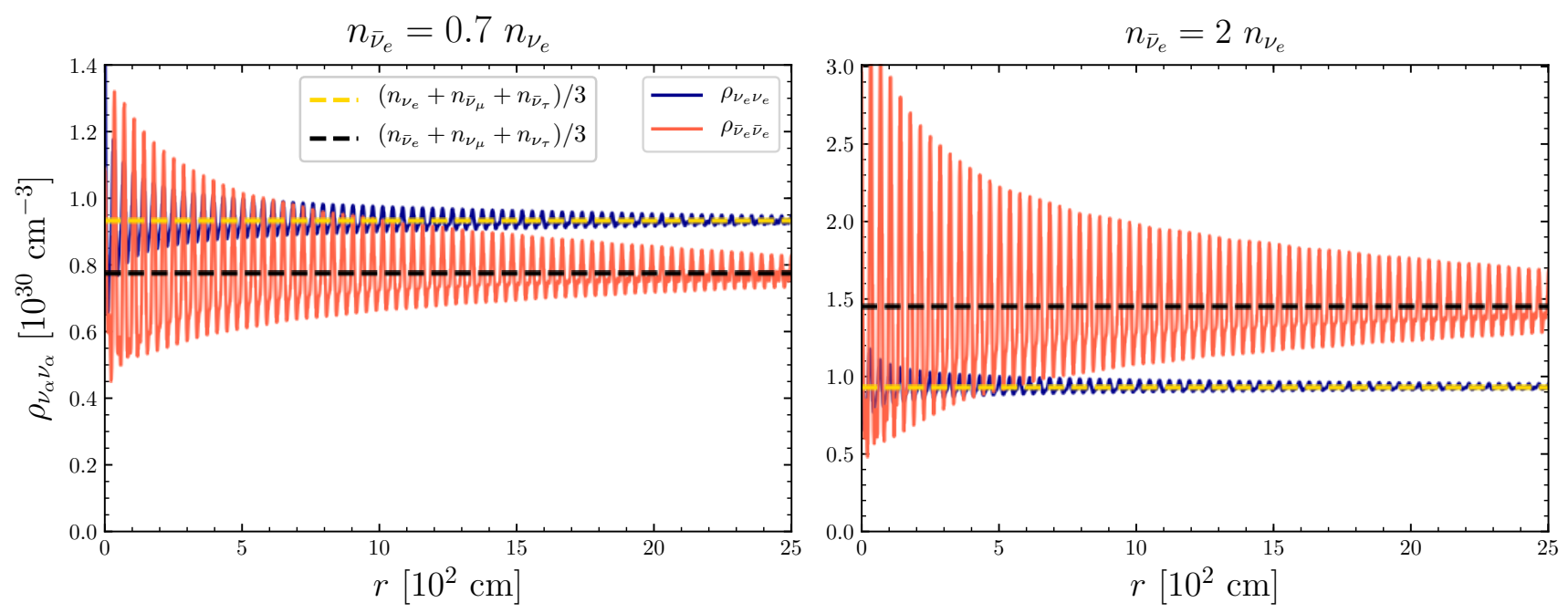

FIG. 3. Angle-averaged survival probabilities of neutrinos and antineutrinos in the three-flavor calculation in the stationary one-dimensional model with $\zeta=10^{3}, \Omega=10 \zeta$ and two different values of $n_{\bar{\nu}_{e}} / n_{\nu_{e}}$. A similar flavor equilibrium can occur among the coupled neutrino/antineutrino species. $\Omega_{\alpha \beta}$ 's are assumed to be equal here.

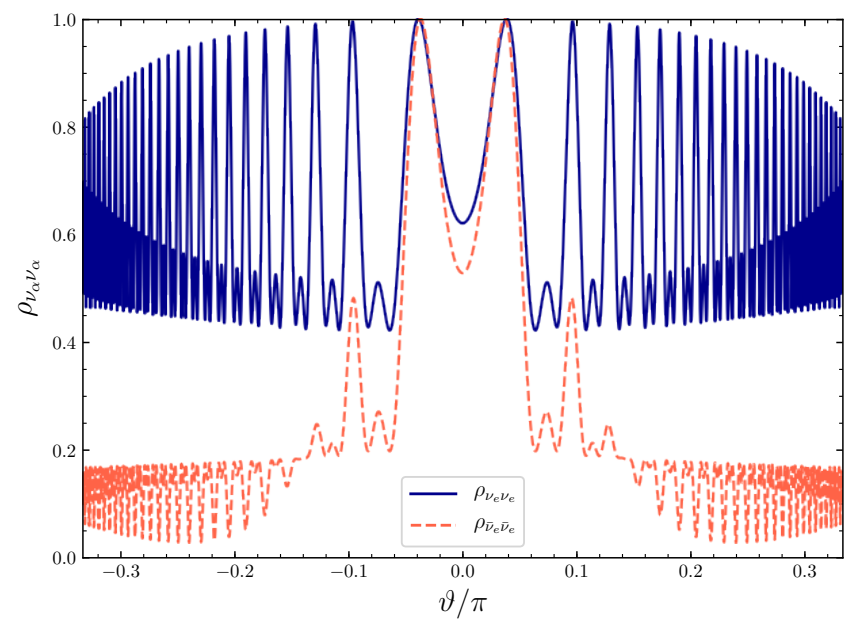

FIG. 4. Angular distributions of the survival probabilities of neutrinos and antineutrinos corresponding to the calculation in Fig 3 with $n_{\bar{\nu}_{e}} / n_{\nu_{e}}=0.7$, and at $r=1000 \mathrm{~cm}$.

$n_{\nu_{\mu}}=n_{\bar{\nu}_{\mu}}=n_{\nu_{\tau}}=n_{\bar{\nu}_{\tau}}$, we observed that $\nu_{e}$ and $\bar{\nu}_{e}$ reach a flavor equilibrium in which

$$
\begin{aligned}
\rho_{\nu_{e} \nu_{e}} & \simeq \frac{n_{\nu_{e}}+n_{\bar{\nu}_{\mu}}+n_{\bar{\nu}_{\tau}}}{3} \\
\rho_{\bar{\nu}_{e} \bar{\nu}_{e}} & \simeq \frac{n_{\bar{\nu}_{e}}+n_{\nu_{\mu}}+n_{\nu_{\tau}}}{3} .
\end{aligned}
$$

This can be explained by noting that the magnetic term couples $\nu_{e}$ to $\bar{\nu}_{\mu}, \bar{\nu}_{\tau}$ and $\bar{\nu}_{e}$ to $\nu_{\mu}, \nu_{\tau}$. In general, the equilibrium values of different neutrino species are only functions of the neutrino number densities but independent of other quantities such as the mass term in the Hamiltonian, $\vartheta_{\max }$, the matter density and so on.
Although individual neutrino (angle) beams can experience large amplitude flavor oscillations, the rapid variations of the angular distributions of neutrino survival probabilities, as shown in Fig. 4, allow neutrinos to reach a flavor equilibrium with relatively small amplitude oscillations around the equilibrium value.

\section{CONCLUSION}

We have studied collective oscillations of Majorana neutrinos in a dense neutrino gas in the presence of strong magnetic fields. Such physical environment is thought to exist in NSM remnants and magneto-rotational CCSNe.

Collective oscillations of Majorana neutrinos can lead to a sort of approximate flavor equilibrium in the presence of strong magnetic field provided that the neutrino transition magnetic moment is strong enough, i.e. when $\Omega=\mu_{\nu} B_{\mathrm{T}}$ is comparable to the other terms in the Hamiltonian. The equilibrium state is determined by the number densities of the coupled (through the magnetic term) neutrino/antineutrino species.

In the presence of nonzero Majorana neutrino magnetic moment, although the total number of neutrinos plus antineutrinos is conserved, the number of neutrinos and antineutrinos are not individually conserved. This is different from the case of usual collective neutrino oscillations in the absence of magnetic fields (and other beyond SM terms). We do not consider the case of Dirac neutrinos in this study where the total number of active neutrinos can also be changed due to the possibility of transition between active and sterile neutrinos.

In our calculations, the magnetic coupling term can play a noticeable role only if $\Omega$ is not much smaller than the other terms in the Hamiltonian. This is different 
from the observation of Refs. [78, 79] where the presence of the magnetic term can significantly modify collective neutrino oscillations even if it is many orders of magnitude smaller than the other terms in the Hamiltonian. This means that one should observe a remarkable impact from the magnetic term even if the length scale associated with $\Omega$ is orders of magnitude larger than the size of the $\mathrm{SN}^{5}$. Note, however, that any comparison between the results presented here and the ones in Refs. [78, 79] must be made with great caution since the employed models are different in several respects.

Apart from providing an example of a physical situation in which collective neutrino oscillations can lead to generic flavor equilibrium, our findings provide useful insight on how the presence of strong lepton number violating channels can impact collective neutrino oscilla- tions. Our results can have important implications for the physics of the most extreme astrophysical environments such as NSM remnants and magneto-rotational CCSNe.

\section{ACKNOWLEDGMENTS}

I would like to thank C. Volpe, S. Shalgar and M. Obergaulinger for valuable discussions and $\mathrm{H}$. Duan for insightful conversations and his helpful comments on the manuscript. I am also grateful to V. Cirigliano for providing me with his notes on neutrino quantum kinetics. This work is partially supported by Physique fondamentale et ondes gravitationelles (PhysFOG) of the Observatoire de Paris.
[1] S. A. Colgate, R. H. White, The Hydrodynamic Behavior of Supernovae Explosions, Astrophys. J. 143 (1966) 626. doi:10.1086/148549.

[2] H. A. Bethe, J. R. Wilson, Revival of a stalled supernova shock by neutrino heating, Astrophys. J. 295 (1985) 1423. doi: $10.1086 / 163343$.

[3] H.-T. Janka, Explosion Mechanisms of CoreCollapse Supernovae, Ann. Rev. Nucl. Part. Sci. 62 (2012) 407-451. arXiv:1206.2503, doi:10.1146/annurev-nucl-102711-094901.

[4] A. Burrows, Colloquium: Perspectives on core-collapse supernova theory, Rev. Mod. Phys. 85 (2013) 245. arXiv: 1210.4921, doi:10.1103/RevModPhys.85.245.

[5] Y. Z. Qian, S. E. Woosley, Nucleosynthesis in neutrino driven winds: 1. The Physical conditions, Astrophys. J. 471 (1996) 331-351. arXiv:astro-ph/9611094, doi:10. $1086 / 177973$.

[6] S. Pastor, G. Raffelt, Flavor oscillations in the supernova hot bubble region: Nonlinear effects of neutrino background, Phys. Rev. Lett. 89 (2002) 191101. arXiv: astro-ph/0207281.

[7] H. Duan, G. M. Fuller, J. Carlson, Y.-Z. Qian, Simulation of Coherent Non-Linear Neutrino Flavor Transformation in the Supernova Environment. 1. Correlated Neutrino Trajectories, Phys. Rev. D74 (2006) 105014. arXiv: astro-ph/0606616, doi:10.1103/PhysRevD.74.105014.

[8] H. Duan, G. M. Fuller, J. Carlson, Y.-Z. Qian, Coherent Development of Neutrino Flavor in the Supernova Environment, Phys. Rev. Lett. 97 (2006) 241101.

\footnotetext{
${ }^{5}$ In the case of usual collective neutrino oscillations (in the absence of magnetic fields), the presence of the off-diagonal term can have a switch-on effect on flavor conversion. Thus, even an infinitesimal nonzero off-diagonal term $\left(\theta_{\mathrm{v}} \ll 1\right)$ can significantly affect flavor evolution of neutrinos. This arises from the flavor instabilities induced by neutrino-neutrino interactions. However, one should not generally expect a similar effect from small $\nu-\bar{\nu}$ transition amplitude due to the presence of $\Omega$ unless neutrinoneutrino interaction has some contribution to $\nu-\bar{\nu}$ transition, as in Refs. [78, 79].
}

arXiv:astro-ph/0608050, doi:10.1103/PhysRevLett. 97.241101.

[9] H. Duan, G. M. Fuller, Y.-Z. Qian, Collective Neutrino Oscillations, Ann. Rev. Nucl. Part. Sci. 60 (2010) 569594. arXiv:1001.2799, doi:10.1146/annurev.nucl. 012809.104524.

[10] S. Chakraborty, R. Hansen, I. Izaguirre, G. Raffelt, Collective neutrino flavor conversion: Recent developments, Nucl. Phys. B908 (2016) 366-381. arXiv:1602.02766, doi:10.1016/j.nuclphysb.2016.02.012.

[11] H. Duan, G. M. Fuller, J. Carlson, Y.-Z. Qian, Flavor Evolution of the Neutronization Neutrino Burst from an O-Ne-Mg Core-Collapse Supernova, Phys. Rev. Lett. 100 (2008) 021101. arXiv:0710.1271, doi:10.1103/ PhysRevLett.100.021101.

[12] B. Dasgupta, A. Dighe, G. G. Raffelt, A. Y. Smirnov, Multiple Spectral Splits of Supernova Neutrinos, Phys. Rev. Lett. 103 (2009) 051105. arXiv:0904.3542, doi: 10.1103/PhysRevLett.103.051105.

[13] S. Galais, C. Volpe, The neutrino spectral split in corecollapse supernovae: a magnetic resonance phenomenon, Phys. Rev. D84 (2011) 085005. arXiv:1103.5302, doi: 10.1103/PhysRevD .84.085005.

[14] H. Duan, G. M. Fuller, J. Carlson, Y.-Z. Qian, Neutrino Mass Hierarchy and Stepwise Spectral Swapping of Supernova Neutrino Flavors, Phys. Rev. Lett. 99 (2007) 241802. arXiv:0707.0290, doi:10.1103/PhysRevLett. 99. 241802 .

[15] H. Duan, Collective neutrino oscillations and spontaneous symmetry breaking, Int. J. Mod. Phys. E24 (09) (2015) 1541008. arXiv:1506.08629, doi:10.1142/ S0218301315410086.

[16] A. Malkus, J. P. Kneller, G. C. McLaughlin, R. Surman, Neutrino oscillations above black hole accretion disks: disks with electron-flavor emission, Phys. Rev. D86 (2012) 085015. arXiv:1207.6648, doi:10.1103/ PhysRevD.86.085015.

[17] A. Malkus, A. Friedland, G. C. McLaughlin, MatterNeutrino Resonance Above Merging Compact ObjectsarXiv: 1403.5797.

[18] A. Malkus, G. C. McLaughlin, R. Surman, Symmetric and Standard Matter-Neutrino Resonances Above Merg- 
ing Compact Objects, Phys. Rev. D93 (4) (2016) 045021. arXiv:1507.00946, doi:10.1103/PhysRevD.93.045021.

[19] A. Vlasenko, G. C. McLaughlin, Matter-neutrino resonance in a multiangle neutrino bulb model, Phys. Rev. D97 (8) (2018) 083011. arXiv:1801.07813, doi:10. 1103/PhysRevD . 97.083011.

[20] S. Shalgar, Multi-angle calculation of the matterneutrino resonance near an accretion disk, JCAP 1802 (02) (2018) 010. arXiv:1707.07692, doi:10.1088/ 1475-7516/2018/02/010.

[21] J. Y. Tian, A. V. Patwardhan, G. M. Fuller, Neutrino Flavor Evolution in Neutron Star Mergers, Phys. Rev. D96 (4) (2017) 043001. arXiv:1703.03039, doi: 10.1103/PhysRevD.96.043001.

[22] M. Frensel, M.-R. Wu, C. Volpe, A. Perego, Neutrino Flavor Evolution in Binary Neutron Star Merger Remnants, Phys. Rev. D95 (2) (2017) 023011. arXiv:1607.05938, doi:10.1103/PhysRevD.95.023011.

[23] Y.-L. Zhu, A. Perego, G. C. McLaughlin, Matter Neutrino Resonance Transitions above a Neutron Star Merger Remnant, Phys. Rev. D94 (10) (2016) 105006. arXiv:1607.04671, doi:10.1103/PhysRevD.94.105006.

[24] M.-R. Wu, H. Duan, Y.-Z. Qian, Physics of neutrino flavor transformation through matterneutrino resonances, Phys. Lett. B752 (2016) 89-94. arXiv:1509.08975, doi: $10.1016 / j$. physletb.2015.11.027.

[25] A. Chatelain, C. Volpe, Helicity coherence in binary neutron star mergers and non-linear feedback, Phys. Rev. D95 (4) (2017) 043005. arXiv:1611.01862, doi: 10.1103/PhysRevD.95.043005.

[26] A. Chatelain, M. C. Volpe, Neutrino propagation in binary neutron star mergers in presence of nonstandard interactions, Phys. Rev. D97 (2) (2018) 023014. arXiv: 1710.11518, doi:10.1103/PhysRevD.97.023014.

[27] M. Delfan Azari, S. Yamada, T. Morinaga, H. Nagakura, S. Furusawa, A. Harada, H. Okawa, W. Iwakami, K. Sumiyoshi, Fast collective neutrino oscillations inside the neutrino sphere in core-collapse supernovaearXiv: 1910.06176.

[28] S. Abbar, H. Duan, K. Sumiyoshi, T. Takiwaki, M. C. Volpe, Fast Neutrino Flavor Conversion Modes in Multidimensional Core-collapse Supernova Models: the Role of the Asymmetric Neutrino DistributionsarXiv:1911. 01983.

[29] R. Glas, H. T. Janka, F. Capozzi, M. Sen, B. Dasgupta, A. Mirizzi, G. Sigl, Fast Neutrino Flavor Instability in the Neutron-star Convection Layer of Three-dimensional Supernova ModelsarXiv:1912.00274.

[30] G. Raffelt, S. Sarikas, D. d. S. Seixas, Axial symmetry breaking in self-induced flavor conversion of supernova neutrino fluxes, Phys. Rev. Lett. 111 (2013) 091101. arXiv:1305.7140, doi:10.1103/PhysRevLett. 111.091101.

[31] H. Duan, Flavor Oscillation Modes In Dense Neutrino Media, Phys. Rev. D88 (2013) 125008. arXiv:1309.7377, doi:10.1103/PhysRevD.88.125008.

[32] H. Duan, S. Shalgar, Flavor instabilities in the neutrino line model, Phys. Lett. B747 (2015) 139-143. arXiv: 1412.7097, doi:10.1016/j.physletb.2015.05.057.

[33] S. Abbar, H. Duan, S. Shalgar, Flavor instabilities in the multiangle neutrino line model, Phys. Rev. D92 (6) (2015) 065019. arXiv:1507.08992, doi:10. 1103/PhysRevD.92.065019.
[34] S. Abbar, H. Duan, Neutrino flavor instabilities in a timedependent supernova model, Phys. Lett. B751 (2015) 4347. arXiv:1509.01538, doi:10.1016/j .physletb. 2015. 10.019.

[35] S. Chakraborty, R. S. Hansen, I. Izaguirre, G. Raffelt, Self-induced flavor conversion of supernova neutrinos on small scales, JCAP 1601 (01) (2016) 028. arXiv:1507. 07569, doi:10.1088/1475-7516/2016/01/028.

[36] B. Dasgupta, A. Mirizzi, Temporal Instability Enables Neutrino Flavor Conversions Deep Inside Supernovae, Phys. Rev. D92 (12) (2015) 125030. arXiv:1509.03171, doi:10.1103/PhysRevD.92.125030.

[37] A. Mirizzi, G. Mangano, N. Saviano, Self-induced flavor instabilities of a dense neutrino stream in a twodimensional model, Phys. Rev. D92 (2) (2015) 021702. arXiv:1503.03485, doi:10.1103/PhysRevD.92.021702.

[38] J. D. Martin, C. Yi, H. Duan, Dynamic fast flavor oscillation waves in dense neutrino gases, Phys. Lett. B800 (2020) 135088. arXiv:1909.05225, doi:10.1016/ j.physletb.2019.135088.

[39] J. D. Martin, S. Abbar, H. Duan, Nonlinear flavor development of a two-dimensional neutrino gas, Phys. Rev. D100 (2) (2019) 023016. arXiv:1904.08877, doi:10. 1103/PhysRevD.100.023016.

[40] R. F. Sawyer, Speed-up of neutrino transformations in a supernova environment, Phys. Rev. D72 (2005) 045003. arXiv:hep-ph/0503013, doi:10.1103/ PhysRevD.72.045003.

[41] R. F. Sawyer, Neutrino cloud instabilities just above the neutrino sphere of a supernova, Phys. Rev. Lett. 116 (8) (2016) 081101. arXiv:1509.03323, doi:10.1103/ PhysRevLett.116.081101.

[42] S. Chakraborty, R. S. Hansen, I. Izaguirre, G. Raffelt, Self-induced neutrino flavor conversion without flavor mixing, JCAP 1603 (03) (2016) 042. arXiv:1602.00698, doi:10.1088/1475-7516/2016/03/042.

[43] I. Izaguirre, G. Raffelt, I. Tamborra, Fast Pairwise Conversion of Supernova Neutrinos: A DispersionRelation Approach, Phys. Rev. Lett. 118 (2) (2017) 021101. arXiv:1610.01612, doi:10.1103/PhysRevLett. 118.021101.

[44] M.-R. Wu, I. Tamborra, Fast neutrino conversions: Ubiquitous in compact binary merger remnants, Phys. Rev. D95 (10) (2017) 103007. arXiv:1701.06580, doi:10. 1103/PhysRevD.95.103007.

[45] F. Capozzi, B. Dasgupta, E. Lisi, A. Marrone, A. Mirizzi, Fast flavor conversions of supernova neutrinos: Classifying instabilities via dispersion relations, Phys. Rev. D96 (4) (2017) 043016. arXiv:1706.03360, doi: 10.1103/PhysRevD.96.043016.

[46] S. A. Richers, G. C. McLaughlin, J. P. Kneller, A. Vlasenko, Neutrino Quantum Kinetics in Compact Objects, Phys. Rev. D99 (12) (2019) 123014. arXiv: 1903.00022, doi:10.1103/PhysRevD.99.123014.

[47] B. Dasgupta, A. Mirizzi, M. Sen, Fast neutrino flavor conversions near the supernova core with realistic flavordependent angular distributions, JCAP 1702 (02) (2017) 019. arXiv: 1609.00528, doi:10.1088/1475-7516/2017/ 02/019.

[48] S. Abbar, H. Duan, Fast neutrino flavor conversion: roles of dense matter and spectrum crossing, Phys. Rev. D98 (4) (2018) 043014. arXiv:1712.07013, doi:10. 1103/PhysRevD.98.043014. 
[49] S. Abbar, M. C. Volpe, On Fast Neutrino Flavor Conversion Modes in the Nonlinear Regime, Phys. Lett. B790 (2019) 545-550. arXiv:1811.04215, doi:10.1016/ j.physletb.2019.02.002.

[50] F. Capozzi, B. Dasgupta, A. Mirizzi, M. Sen, G. Sigl, Collisional triggering of fast flavor conversions of supernova neutrinos, Phys. Rev. Lett. 122 (9) (2019) 091101. arXiv: 1808.06618, doi:10.1103/PhysRevLett.122.091101.

[51] F. Capozzi, G. Raffelt, T. Stirner, Fast Neutrino Flavor Conversion: Collective Motion vs. Decoherence, JCAP 1909 (2019) 002. arXiv:1906.08794, doi:10.1088/ $1475-7516 / 2019 / 09 / 002$.

[52] C. Dring, R. S. L. Hansen, M. Lindner, Stability of three neutrino flavor conversion in supernovae, JCAP 1908 (2019) 003. arXiv:1905.03647, doi:10.1088/ 1475-7516/2019/08/003.

[53] M. Chakraborty, S. Chakraborty, Three flavor neutrino conversions in supernovae: Slow \& Fast instabilitiesarXiv: 1909.10420.

[54] L. Johns, H. Nagakura, G. M. Fuller, A. Burrows, Neutrino oscillations in supernovae: angular moments and fast instabilitiesarXiv: 1910.05682.

[55] S. Shalgar, I. Padilla-Gay, I. Tamborra, Neutrino propagation hinders fast pairwise flavor conversionsarXiv: 1911.09110.

[56] J. F. Cherry, G. M. Fuller, S. Horiuchi, K. Kotake, T. Takiwaki, T. Fischer, Time of Flight and Supernova Progenitor Effects on the Neutrino HaloarXiv: 1912.11489.

[57] C. Giunti, A. Studenikin, Neutrino electromagnetic properties, Phys. Atom. Nucl. 72 (2009) 2089-2125. arXiv: 0812.3646, doi:10.1134/S1063778809120126.

[58] C. Broggini, C. Giunti, A. Studenikin, Electromagnetic Properties of Neutrinos, Adv. High Energy Phys. 2012 (2012) 459526. arXiv:1207.3980, doi:10.1155/2012/ 459526.

[59] A. Studenikin, Status and perspectives of neutrino magnetic moments, J. Phys. Conf. Ser. 718 (6) (2016) 062076. arXiv:1603.00337, doi:10.1088/1742-6596/ 718/6/062076.

[60] P. Msta, C. D. Ott, D. Radice, L. F. Roberts, E. Schnetter, R. Haas, A large scale dynamo and magnetoturbulence in rapidly rotating core-collapse supernovae, Nature 528 (2015) 376. arXiv:1512.00838, doi:10.1038/ nature15755.

[61] K. Fujikawa, R. Shrock, The Magnetic Moment of a Massive Neutrino and Neutrino Spin Rotation, Phys. Rev. Lett. 45 (1980) 963. doi:10.1103/PhysRevLett.45.963.

[62] M. Lindner, B. Radovi, J. Welter, Revisiting Large Neutrino Magnetic Moments, JHEP 07 (2017) 139. arXiv: 1706.02555, doi:10.1007/JHEP07 (2017) 139.

[63] N. F. Bell, V. Cirigliano, M. J. Ramsey-Musolf, P. Vogel, M. B. Wise, How magnetic is the Dirac neutrino?, Phys. Rev. Lett. 95 (2005) 151802. arXiv:hep-ph/0504134, doi:10.1103/PhysRevLett.95.151802.

[64] S. Davidson, M. Gorbahn, A. Santamaria, From transition magnetic moments to majorana neutrino masses, Phys. Lett. B626 (2005) 151-160. arXiv:hep-ph/ 0506085, doi:10.1016/j.physletb.2005.08.086.

[65] B. C. Canas, O. G. Miranda, A. Parada, M. Tortola, J. W. F. Valle, Updating neutrino magnetic moment constraints, Phys. Lett. B753 (2016) 191-198, [Addendum: Phys. Lett.B757,568(2016)]. arXiv:1510.01684, doi:10.1016/j.physletb.2016.03.
078, 10.1016/j.physletb. 2015.12.011.

[66] A. G. Beda, V. B. Brudanin, V. G. Egorov, D. V. Medvedev, V. S. Pogosov, M. V. Shirchenko, A. S. Starostin, The results of search for the neutrino magnetic moment in GEMMA experiment, Adv. High Energy Phys. 2012 (2012) 350150. doi:10.1155/2012/350150.

[67] E. W. Kolb, D. L. Tubbs, D. A. Dicus, Lepton Number Violation, Majorana Neutrinos, and Supernovae, Astrophys. J. 255 (1982) L57. doi:10.1086/183769.

[68] J. Schechter, J. W. F. Valle, Majorana Neutrinos and Magnetic Fields, Phys. Rev. D24 (1981) 1883-1889, [Erratum: Phys. Rev.D25,283(1982)]. doi:10.1103/ PhysRevD.25.283, 10.1103/PhysRevD.24.1883.

[69] C.-S. Lim, W. J. Marciano, Resonant Spin - Flavor Precession of Solar and Supernova Neutrinos, Phys. Rev. D37 (1988) 1368-1373, [,351(1987)]. doi:10.1103/ PhysRevD.37.1368.

[70] A. B. Balantekin, P. J. Hatchell, F. Loreti, Matter Enhanced Spin Flavor Precession of Solar Neutrinos With Transition Magnetic Moments, Phys. Rev. D41 (1990) 3583. doi:10.1103/PhysRevD.41.3583.

[71] E. K. Akhmedov, Z. G. Berezhiani, Implications of Majorana neutrino transition magnetic moments for neutrino signals from supernovae, Nucl. Phys. B373 (1992) 479497. doi : 10.1016/0550-3213 (92) 90441-D.

[72] E. K. Akhmedov, A. Lanza, D. W. Sciama, Resonant spin flavor precession of neutrinos and pulsar velocities, Phys. Rev. D56 (1997) 6117-6124. arXiv:hep-ph/9702436, doi:10.1103/PhysRevD.56.6117.

[73] S. Ando, K. Sato, Three generation study of neutrino spin flavor conversion in supernova and implication for neutrino magnetic moment, Phys. Rev. D67 (2003) 023004. arXiv:hep-ph/0211053, doi:10.1103/ PhysRevD.67.023004.

[74] O. Lychkovskiy, S. Blinnikov, Spin flip of neutrinos with magnetic moment in core-collapse supernova, Phys. Atom. Nucl. 73 (2010) 614-624. arXiv:0905.3658, doi: 10.1134/S106377881004006X.

[75] A. B. Balantekin, C. Volpe, J. Welzel, Impact of the Neutrino Magnetic Moment on Supernova r-process Nucleosynthesis, JCAP 0709 (2007) 016. arXiv:0706.3023, doi:10.1088/1475-7516/2007/09/016.

[76] A. V. Kuznetsov, N. V. Mikheev, A. A. Okrugin, DiracNeutrino Magnetic Moment and the Dynamics of a Supernova Explosion, JETP Lett. 89 (2009) 97-101. arXiv : 0903.2321, doi:10.1134/S0021364009030011.

[77] E. K. Akhmedov, T. Fukuyama, Supernova prompt neutronization neutrinos and neutrino magnetic moments, JCAP 0312 (2003) 007. arXiv:hep-ph/0310119, doi: 10.1088/1475-7516/2003/12/007.

[78] A. de Gouvea, S. Shalgar, Effect of Transition Magnetic Moments on Collective Supernova Neutrino Oscillations, JCAP 1210 (2012) 027. arXiv:1207.0516, doi:10.1088/ 1475-7516/2012/10/027.

[79] A. de Gouvea, S. Shalgar, Transition Magnetic Moments and Collective Neutrino Oscillations:Three-Flavor Effects and Detectability, JCAP 1304 (2013) 018. arXiv : 1301.5637, doi:10.1088/1475-7516/2013/04/018.

[80] M. Dvornikov, Evolution of a dense neutrino gas in matter and electromagnetic field, Nucl. Phys. B855 (2012) 760-773. arXiv:1108.5043, doi:10.1016/j.nuclphysb. 2011.10 .025$.

[81] A. Dobrynina, A. Kartavtsev, G. Raffelt, Helicity oscillations of Dirac and Majorana neutrinos, Phys. Rev. 
D93 (12) (2016) 125030. arXiv:1605.04512, doi:10. 1103/PhysRevD.93.125030.

[82] P. Kurashvili, K. A. Kouzakov, L. Chotorlishvili, A. I. Studenikin, Spin-flavor oscillations of ultrahigh-energy cosmic neutrinos in interstellar space: The role of neutrino magnetic moments, Phys. Rev. D96 (10) (2017) 103017. arXiv:1711.04303, doi:10.1103/PhysRevD.96. 103017.

[83] G. Sigl, G. Raffelt, General kinetic description of relativistic mixed neutrinos, Nucl. Phys. B406 (1993) 423451. doi: 10 . 1016/0550-3213(93) 90175-0.

[84] P. Strack, A. Burrows, A generalized boltzmann formalism for oscillating neutrinos, Phys. Rev. D71 (2005) 093004. arXiv:hep-ph/0504035.

[85] C. Y. Cardall, Liouville equations for neutrino distribution matrices, Phys. Rev. D78 (2008) 085017. arXiv: 0712.1188, doi:10.1103/PhysRevD.78.085017.

[86] C. Volpe, D. Vnnen, C. Espinoza, Extended evolution equations for neutrino propagation in astrophysical and cosmological environments, Phys. Rev. D87 (11) (2013) 113010. arXiv:1302.2374, doi:10.1103/PhysRevD.87. 113010.

[87] A. Vlasenko, G. M. Fuller, V. Cirigliano, Neutrino Quantum Kinetics, Phys. Rev. D89 (10) (2014) 105004. arXiv: 1309.2628, doi:10.1103/PhysRevD.89.105004.

[88] V. Cirigliano, G. M. Fuller, A. Vlasenko, A New Spin on Neutrino Quantum Kinetics, Phys. Lett. B747 (2015) 2735. arXiv:1406.5558, doi:10.1016/j.physletb. 2015. 04.066.

[89] D. Vnnen, C. Volpe, Linearizing neutrino evolution equations including neutrino-antineutrino pairing correlations, Phys. Rev. D88 (2013) 065003. arXiv:1306.6372, doi:10.1103/PhysRevD.88.065003.

[90] C. Volpe, Neutrino Quantum Kinetic Equations, Int. J. Mod. Phys. E24 (09) (2015) 1541009. arXiv:1506.06222, doi:10.1142/S0218301315410098.

[91] M. Tanabashi, et al., Review of Particle Physics, Phys. Rev. D98 (3) (2018) 030001. doi:10.1103/PhysRevD.98. 030001. 